\section{Tendência de mortalidade por câncer de colo de útero para o Estado de Minas Gerais, Brasil, 1980-2005}

\author{
Cervical cancer mortality trends in Minas Gerais \\ State, Brazil, 1980-2005
}

\footnotetext{
${ }_{1}$ Centro de Tratamento de Neoplasias, Juiz de Fora, Brasil.

2 Programa de Pós-graduação em Saúde Coletiva Universidade Federal de Juiz de Fora, Juiz de Fora, Brasil. 3 Faculdade de Medicina, Universidade Federal de Juiz de Fora, Juiz de Fora, Brasil. ${ }^{4}$ Instituto de Ciências Exatas, Universidade Federal de Juiz de Fora, Juiz de Fora, Brasil.

Correspondência C. M. M. Alves Centro de Tratamento de Neoplasias.

Rua Espírito Santo 1115, apto. 1809, Juiz de Fora, MG 36016-905, Brasil.

c.meurer@terra.com.br
}

\begin{abstract}
This study evaluated the cervical cancer mortality trend from 1980 to 2005 in the State of Minas Gerais, Brazil. The demographic and mortality data available on the website of the Statistics Department of the Unified $\mathrm{Na}$ tional Health System (DATASUS) were used to estimate the mortality rate for uterine cervical cancer and unspecified uterine cancer for the State during the period. Linear regression was performed, with mortality rate as the response variable and year of diagnosis as the explanatory variable. Mortality varied from 9.18/100,000 in 1980 to 5.70/100,000 in 2005. The log-linear model showed a 1.93\% annual reduction in mortality due to cervical cancer for the State during the study period ( $p=$ 0.000), and this drop was observed mainly in cases classified as unspecified uterine cancer. The downward mortality trend observed for this disease suggests improved access to timely and adequate diagnosis and treatment in Minas Gerais in recent years, although coverage of screening is still unsatisfactory.
\end{abstract}

Uterine Cervical Neoplasms; Women's Health; Mortality
Christiane Maria Meurer Alves 1,2

Maximiliano Ribeiro Guerra ${ }^{3}$

Ronaldo Rocha Bastos 4

\section{Introdução}

Apesar da possibilidade de detecção precoce por meio de exame de rastreamento populacional reconhecido, o câncer de colo uterino é ainda o segundo câncer mais comum entre mulheres, e foi responsável por mais de $250 \mathrm{mil}$ mortes no mundo em 2005, sendo que cerca de $80 \%$ dos casos ocorrem em países em desenvolvimento 1,2 , onde a maioria dos casos foi diagnosticada como doença localmente avançada ou metastática 3 .

Tem sido observada uma importante redução da mortalidade por esta neoplasia nas últimas décadas em países desenvolvidos, especialmente após a introdução dos programas de rastreamento da doença nas décadas de 1960 e 1970 2. Estudos mostram tal redução em países europeus como Finlândia 4, Islândia 5, Bélgica 6,7, Escócia 8 e Reino Unido ${ }^{9}$, contudo, tem sido observado um aumento na sua incidência nas faixas etárias mais jovens. Na Finlândia, por exemplo, foi evidenciado um decréscimo na mortalidade pela doença de cerca de $80 \%$ no período compreendido entre 1963-1995 4. Em comum, estes países já apresentam um programa de rastreamento organizado há pelo menos três décadas.

Nos Estados Unidos 10 e Canadá 11, foi observada redução da mortalidade por câncer de colo uterino nas últimas décadas, apesar do aumento da incidência do adenocarcinoma de colo uterino 12 . 
No continente asiático, entretanto, a tendência da mortalidade por câncer de colo de útero tem exibido importantes variações geográficas. Na província chinesa de Shandong, houve uma clara redução de mortalidade, de 21,11/100 mil no período de 1970-1974 para 5,57/100 mil no período de 1985-1989 13. Em contraste, em Taiwan, a mortalidade aumentou de 6,06/100 mil em 1974 para 10,02/100 mil em 199314.

Em estudo sobre a mortalidade e incidência de câncer cervical na América Latina, baseado nos dados do GLOBOCAN 2000 15, foram explicitados os obstáculos encontrados para se obter um panorama completamente confiável da doença nestes países, uma vez que, embora muitos dos países já tivessem iniciado seu registro de câncer de base populacional na última década, ainda persistiam dificuldades para a produção de dados com bom nível de qualidade. Além disso, muitos casos de câncer cervical ainda eram apresentados como câncer de útero porção não especificada. Foram evidenciados também indícios de declínio modesto do risco de câncer cervical na maioria dos países da América Latina 16.

No Brasil, ao se agruparem os dados de óbito por câncer de colo de útero e útero porção não especificada no Estado do Rio Grande do Sul para o período de 1979-1995, foi evidenciada estabilidade da mortalidade, diferente do encontrado quando analisados apenas os dados relativos aos óbitos por câncer de colo de útero no mesmo período, os quais demonstraram aumento ${ }^{17}$. Estudo conduzido em Salvador, Bahia, Brasil, mostrou declínio na mortalidade pela doença, tendo incluído também os óbitos por câncer de colo de útero não especificado 18 .

Em estudo publicado sobre a mortalidade por câncer de útero no Município de São Paulo, Brasil, entre 1980-1999, considerando os óbitos por câncer de colo de útero, endométrio e útero porção não especificada, foi constatado que a mortalidade por câncer de colo uterino mantevese estável no período de 1980-1999, com redução na mortalidade por câncer de útero porção não especificada. Argumentou-se que a maioria dos casos apresentados como câncer de útero porção não especificada eram, na realidade, casos de câncer de colo de útero. Esta hipótese foi levantada uma vez que a queda na mortalidade iniciou-se ainda nas faixas etárias mais jovens, onde o câncer de endométrio apresenta incidência praticamente nula, além da ausência de redução observada na faixa etária mais elevada 19.

No Estado de Minas Gerais, Brasil, o câncer foi a segunda causa de morte entre mulheres no ano de 2006, sendo precedido apenas pelas doenças do aparelho circulatório. Entre as neoplasias malignas conhecidas, o câncer de colo de útero apresentou-se como a sexta causa de morte no sexo feminino. Em relação ao número de casos novos, a taxa de incidência ajustada por idade pela população mundial para o ano de 2000 em Belo Horizonte foi de 31,98 casos por 100 mil mulheres para o câncer de colo de útero e de 3,92 casos por 100 mil mulheres para o câncer de útero porção não especificada 20 .

Considerando a magnitude desta neoplasia em termos de incidência e mortalidade e seu conseqüente impacto no setor de saúde brasileiro, apesar dos esforços que têm sido despendidos para o controle efetivo da doença no país, foi realizado um estudo da tendência temporal de mortalidade para o câncer de colo uterino e útero não especificado no Estado de Minas Gerais, no período de 1980-2005.

\section{Material e métodos}

Tratou-se de um estudo de séries temporais, cujos dados demográficos e de mortalidade para câncer de colo de útero e útero porção não especificada relativos ao Estado de Minas Gerais no período de 1980-2005 foram obtidos através da base de dados da página da Internet do Departamento de Informática do SUS (DATASUS, http://www.datasus.gov.br), tomando como base as Informações de Saúde - Demográficas e Socioeconômicas e o Sistema de Informações sobre Mortalidade (SIM), respectivamente.

Optou-se por avaliar também os casos de câncer de útero porção não especificada, uma vez que foi demonstrado que a maioria destes casos é representada por casos de câncer de colo de útero 18.

Por meio do programa Epi Info versão 3.3.2 (Centers for Disease Control and Prevention, Atlanta, Estados Unidos), foram obtidas as freqüências anuais das patologias incluídas no estudo, baseando-se na Classificação Internacional de Doenças (CID). Para o período de 1980-1995, aplicou-se a 9a revisão da CID (CID-9) 21, sendo que, para o restante do período, foi adotada a 10a revisão (CID-10) 22. Na CID-9, os códigos correspondentes eram: 180 para câncer do colo de útero e 179 para câncer de útero porção não especificada e, na CID-10, C53 e C55, respectivamente.

Foram incluídos todos os casos de óbitos em mulheres que tiveram como causa básica o câncer de colo de útero e o câncer de útero porção não especificada.

Os coeficientes de mortalidade brutos e padronizados por 100 mil habitantes, para cada ano, foram calculados pelo método direto 23 , utilizando o Microsoft Excel 2003 (Microsoft Corp., 
Estados Unidos). Tais taxas foram calculadas tanto para o conjunto dos dados quanto para as duas patologias separadamente.

A padronização foi feita tomando como base a população mundial, população de referência também utilizada para a construção dos indicadores disponibilizados a partir dos Registros de Câncer de Base Populacional (RCBP) em funcionamento no Brasil 20.

Para o cálculo da regressão linear, utilizou-se o programa estatístico SPSS 8.0 (SPSS Inc., Chicago, Estados Unidos). Tendo em vista que os coeficientes de mortalidade podem seguir uma distribuição não linear, considera-se o modelo exponencial como o melhor modelo para predizer os coeficientes correspondentes ao ano do óbito ${ }^{24}$. Os coeficientes padronizados de mortalidade para as faixas etárias foram, portanto, log-transformados para a realização do modelo log-linear.

Estimou-se, assim, a variação percentual anual para o período. O procedimento foi realizado por faixa etária e para o total dos casos, considerando os casos codificados como câncer de colo de útero, útero porção não especificada e ambos (Tabela 1).

\section{Resultados}

O estudo incluiu 12.606 casos, sendo que 6.734 (53,42\%) eram casos de câncer de colo de útero e $5.872(46,58 \%)$ eram casos de câncer de útero porção não especificada.

Para os cânceres de colo de útero e de útero porção não especificada, foi observada diminuição gradativa da mortalidade no período estudado, passando de 9,18/100 mil em 1980 para 5,70/100 mil em 2005, sendo também constatada uma redução da mortalidade para cada um dos estratos etários analisados.

Conforme pode ser constatado na Tabela 1, houve uma redução de 1,93\% ao ano nas taxas de mortalidade para as duas neoplasias. Entre todas as faixas etárias, a menor redução foi evidenciada para o grupo de 65-69 anos (1,21\%), sendo esta também significativa $(\mathrm{p}=0,001)$.

Quando considerados apenas os casos codificados como câncer de colo uterino, a redução da mortalidade ao longo do período foi menos expressiva, sendo observada uma diminuição de apenas $0,56 \%$ ao ano no período estudado ( $\mathrm{p}=$ 0,009). Em relação às faixas etárias, a maior redução foi constatada nos grupos etários de 50-54 anos (1,39\%) e de 55-59 anos (1,33\%), sendo que, para as faixas de 60-64, de 65-69 anos e acima de 80 anos, foi evidenciado aumento da mortalidade $(0,05 \%, 0,37 \%$ e $0,15 \%$, respectivamente), embora não significativo $(\mathrm{p}>0,05)$. A redução da mortalidade pela doença observada na população de estudo deveu-se, principalmente, à maior redução dos óbitos com causa básica classificada como câncer de útero porção não especificada (Tabela 1).

Tabela 1

Variação percentual anual estimada para câncer de colo de útero, câncer de útero porção não especificada e ambos, segundo grupos etários, no Estado de Minas Gerais, Brasil, período de 1980-2005.

\begin{tabular}{|c|c|c|c|c|c|c|}
\hline \multirow{2}{*}{$\begin{array}{l}\text { Faixa etária } \\
\text { (anos) }\end{array}$} & \multicolumn{2}{|c|}{ Câncer de colo uterino (C53) * } & \multicolumn{2}{|c|}{ Câncer de útero porção não especificada (C55) * } & \multicolumn{2}{|c|}{$\mathrm{C} 53+\mathrm{C} 55$ * } \\
\hline & Variação \% anual & Valor de p & Variação \% anual & Valor de $p$ & Variação \% anual & Valor de $p$ \\
\hline $0-29$ & - & - & - & - & $-1,68$ & 0,146 \\
\hline $30-34$ & $-0,70$ & 0,547 & $-4,95$ & 0,000 & $-2,39$ & 0,004 \\
\hline $35-39$ & $-0,80$ & 0,249 & $-4,99$ & 0,000 & $-2,30$ & 0,000 \\
\hline $40-44$ & $-0,72$ & 0,248 & $-3,24$ & 0,000 & $-1,63$ & 0,000 \\
\hline $45-49$ & $-0,73$ & 0,240 & $-4,32$ & 0,000 & $-2,16$ & 0,000 \\
\hline $50-54$ & $-1,39$ & 0,009 & $-4,51$ & 0,000 & $-2,80$ & 0,000 \\
\hline $55-59$ & $-1,33$ & 0,048 & $-3,86$ & 0,000 & $-2,46$ & 0,000 \\
\hline $60-64$ & 0,05 & 0,912 & $-4,04$ & 0,000 & $-1,68$ & 0,000 \\
\hline $65-69$ & 0,37 & 0,363 & $-2,72$ & 0,000 & $-1,21$ & 0,001 \\
\hline $70-74$ & $-0,29$ & 0,684 & $-2,95$ & 0,000 & $-1,69$ & 0,000 \\
\hline $75-79$ & $-0,16$ & 0,816 & $-2,46$ & 0,002 & $-1,40$ & 0,003 \\
\hline$>80$ & 0,15 & 0,839 & $-2,75$ & 0,000 & $-1,57$ & 0,000 \\
\hline Total & $-0,56$ & 0,009 & $-3,57$ & 0,000 & $-1,93$ & 0,000 \\
\hline
\end{tabular}

* De acordo com a 10ạ revisão da Classificação Internacional de Doenças (CID-10) 22. 
A Figura 1 exibe a representação gráfica da regressão linear para o total de casos. Nas Figuras 2 e 3, é mostrada a regressão linear para o câncer de colo de útero e para útero porção não especificada, respectivamente. Por meio destas figuras, pode-se visualizar que, apesar de a redução na mortalidade ter sido observada tanto para o conjunto dos dados quanto para cada patologia isoladamente, tal redução foi mais acentuada na mortalidade por câncer de útero porção não especificada.

\section{Discussão e conclusão}

Foi observada uma tendência de redução da mortalidade por câncer cervical de cerca de 1,93\% ao ano para o Estado de Minas Gerais no período compreendido entre 1980-2005. Neste sentido, cabe comentar que estudos têm demonstrado queda das taxas de mortalidade por esta doença ao longo dos anos em diversas regiões 4,5,6,7,8,9, $10,11,13,16,17,18,19$, sugerindo melhora no acesso a métodos de diagnóstico e tratamento oportunos e adequados nos locais e períodos considerados.

No entanto, verifica-se que a referida redução da mortalidade na população de estudo foi mais acentuada para o câncer de útero porção

Figura 1

Regressão linear por câncer de colo uterino e útero porção não especificada, no Estado de Minas Gerais, Brasil, período de 1980-2005

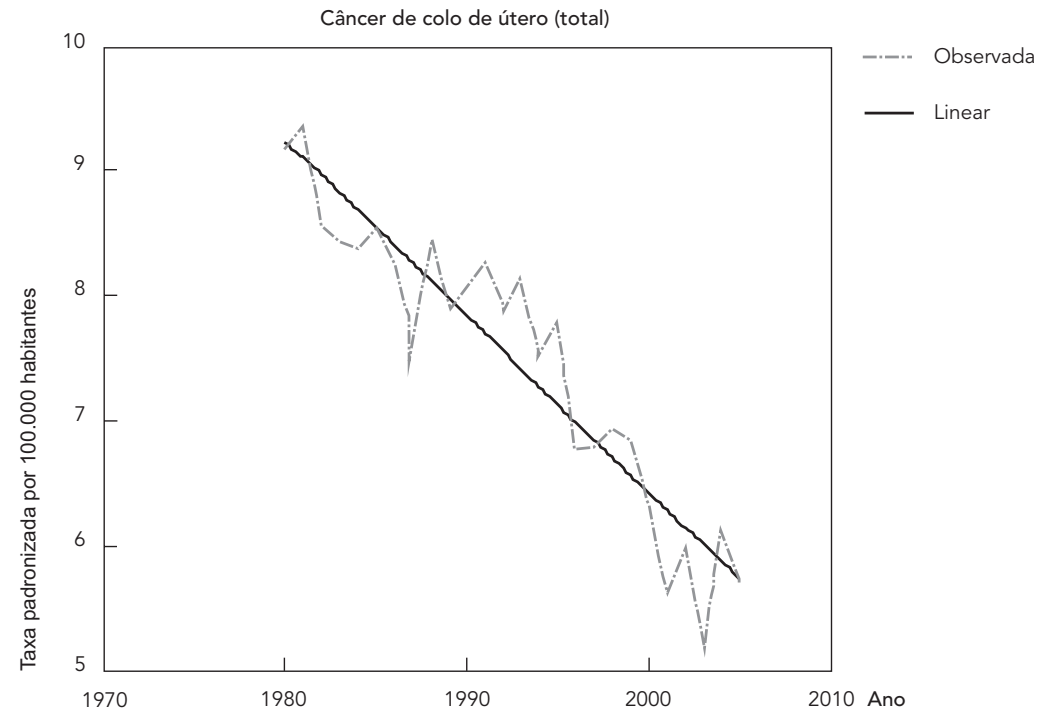

Cad. Saúde Pública, Rio de Janeiro, 25(8):1693-1700, ago, 2009 não especificada, o que também foi constatado por Fonseca et al. 19 em estudo sobre tendência de mortalidade pela doença no Município de São Paulo. Para melhor entendimento deste achado, deve-se levar em consideração que a imprecisão na identificação da localização do tumor, de uma forma geral, pode representar, entre outras possíveis explicações: capacidade diagnóstica precária; óbito sem assistência; doença avançada no momento do diagnóstico, o que torna difícil a identificação de sua topografia primária; e baixa qualidade do registro (codificação da causa básica) no período considerado. Assim sendo, a redução mais expressiva verificada na mortalidade por câncer de útero porção não especificada pode estar relacionada, na região e período avaliados, tanto à melhoria no acesso ao cuidado em saúde quanto à adoção de abordagem terapêutica mais efetiva.

A queda da mortalidade observada em Minas Gerais poderia também estar associada à redução na incidência do câncer cervical neste estado. Todavia, seria necessário um RCBP mais abrangente e com maior período de dados consolidados para confirmar tal possibilidade. Vale enfatizar que existem apenas 22 RCBP implantados no Brasil, quase em sua totalidade, em capitais, sendo que somente 16 destes possuem informações consolidadas, isto é, com pelo menos um ano de informações sobre os casos definitivos (incidência), destacando-se entre os mesmos o RCBP de Belo Horizonte. Por meio de dados do referido registro, foram calculadas as taxas de incidência para Belo Horizonte, ajustadas por idade pela população mundial e relativas apenas ao ano de 2000, tanto do câncer de colo uterino $(31,98 / 100$ mil; valor este relativamente alto, se comparado com as áreas de maior e menor incidência do mundo) 25 , quanto do câncer de útero porção não especificada (3,92/100 mil), não sendo disponibilizados outros dados que possam permitir considerações sobre o comportamento da incidência da doença ao longo do tempo na região considerada 20 .

Percebe-se, assim, a importância da ampliação e sustentabilidade do registro de câncer para possibilitar a melhor compreensão da evolução da doença, contribuindo também para a melhoria da atribuição da causa básica. No tocante a erros de codificação de causa básica, estudo publicado sobre a mortalidade por câncer cervical na Europa demonstrou que, em 1995, países como França e Itália ainda apresentavam proporções superiores a $50 \%$ de casos classificados como câncer de útero porção não especificada 26 . A mesma preocupação foi esboçada por Swerdlow et al. 8 em seu estudo sobre mortalidade e incidência por câncer na Escócia, onde os auto- 
res afirmaram que o comportamento do câncer uterino é potencialmente aberto a artefatos, uma vez que uma parcela dos casos da doença tende a ser classificada como útero porção não especificada. Observaram, ainda, que, para a incidência, esta proporção de imprecisão na classificação foi menor (5,8\%) e não se modificou muito ao longo do tempo e que, para a mortalidade, os números foram mais elevados (19,6\%).

No Brasil, estudos publicados sobre a mortalidade por câncer cervical em Salvador 18, Rio Grande do Sul 17 e São Paulo 19 avaliaram os óbitos por câncer cervical em conjunto (câncer de colo de útero e câncer de útero porção não especificada) e separadamente. No que tange à queda da mortalidade por câncer de útero porção não especificada, os achados deste estudo são semelhantes àqueles encontrados por Fonseca et al. ${ }^{19}$ para o Município de São Paulo, que observaram queda acentuada desde as faixas etárias mais jovens, onde o câncer de endométrio é muito pouco freqüente, o que sugere que tal redução esteve mais relacionada ao câncer de colo de útero. No Estado de Minas Gerais, a queda da mortalidade pela doença também ocorreu nas faixas etárias mais avançadas, entretanto de forma menos expressiva (de $2,4 \%$ a $2,9 \%$ ), o que é condizente com a hipótese de a maioria dos casos classificados de forma imprecisa (câncer de útero porção não especificada) tratam-se de casos de câncer de colo uterino. Esta redução pode estar relacionada à expansão do acesso ao rastreamento colpocitológico, além de maiores informações sobre a doença e sua prevenção, uma vez que a maior precisão na atribuição da causa básica, muito provavelmente, apresentase relacionada à maior disponibilidade de métodos diagnósticos 19.

Neste sentido, vale comentar que o rastreamento citológico do câncer de colo uterino foi iniciado no Brasil no fim da década de 1950 e início dos anos 1960, de forma incipiente e com baixa cobertura, não se constituindo, a princípio, como uma prática coletiva. Em 1983, o Ministério da Saúde organizou o Programa de Assistência Integral à Saúde da Mulher (PAISM) e, com a criação do Sistema Único de Saúde (SUS) a partir da Constituição Federal brasileira em 1988, foram desenvolvidas iniciativas mais amplas voltadas para o combate da doença, sendo instituído, em 1997, o Programa Nacional de Rastreamento do Câncer Cervical, incorporado ao Programa Viva Mulher, com ações direcionadas, especialmente, às mulheres na faixa de 25-59 anos, com ênfase para aquelas com 35-49 anos 27,28. Embora a periodicidade preconizada pelo citado Programa para a realização do exame seja, inicialmente, de um exame por ano e, a partir de dois exames nor-
Figura 2

Regressão linear por câncer de colo uterino, no Estado de Minas Gerais, Brasil, período de 1980-2005.

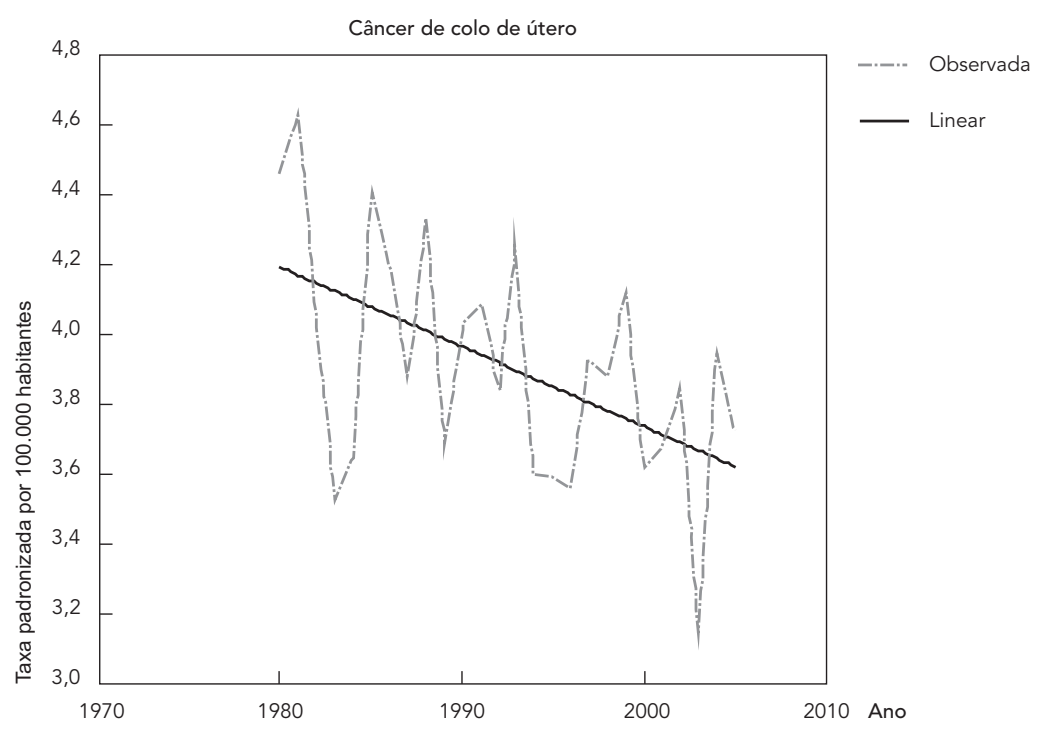

Figura 3

Regressão linear por câncer de útero porção não especificada, no Estado de Minas Gerais, Brasil, período de 1980-2005.

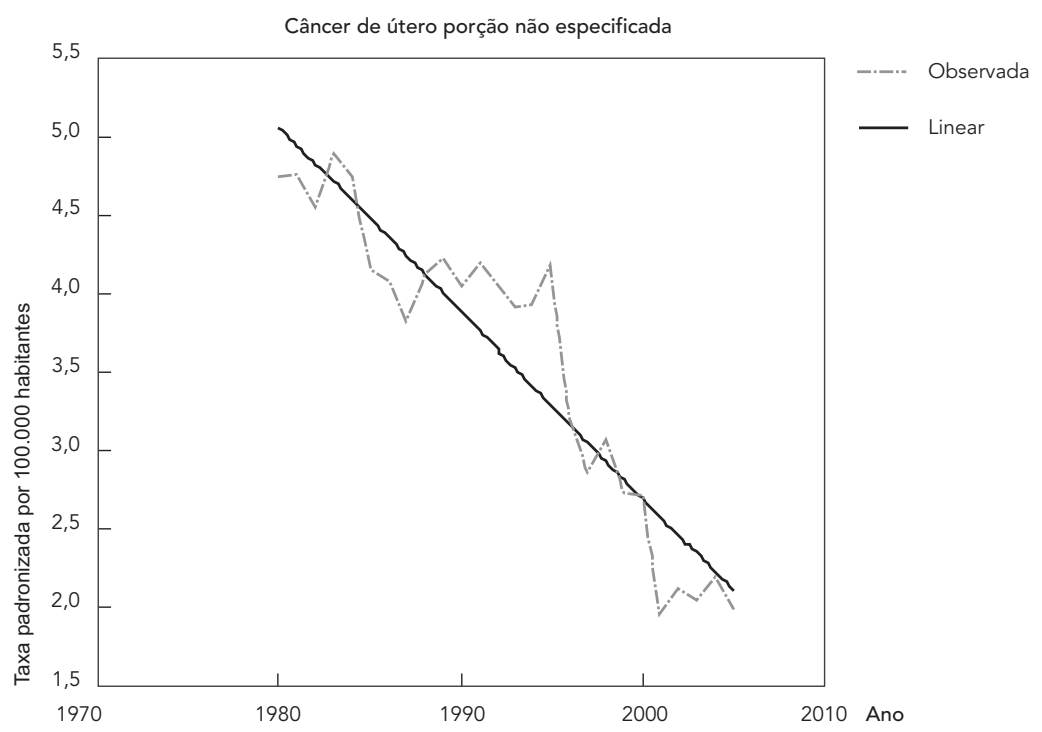


mais consecutivos (com intervalo de um ano), a cada três anos 29 , não foi prevista, entretanto, a convocação/mobilização da população-alvo para a sua realização. Portanto, a precocidade da organização do sistema de saúde brasileiro para o enfrentamento dessa patologia não nos permite atribuir apenas ao rastreamento a redução observada da mortalidade pela doença. Entretanto, deve-se levar em consideração que a incorporação progressiva da prática do exame de Papanicolaou nos serviços de saúde talvez seja um fator muito importante em nosso país, pois sua disponibilidade, mesmo que inicialmente orientada apenas por demanda, provavelmente possibilitou tanto o tratamento de maior número de lesões precursoras quanto a realização de diagnósticos precoces da patologia, aumentando a possibilidade de cura da doença e reduzindo seu efeito na mortalidade 17,18,19.

Vale mencionar também que, no Estado de Minas Gerais, a implementação do Programa Nacional de Controle do Câncer do Colo do Útero - Viva Mulher, a partir do final da década de 90, possibilitou a implantação do Sistema de Informação do Câncer de Colo Uterino (SISCOLO), importante instrumento de avaliação e planejamento, e a intensificação da capacitação específica para o exame preventivo de Papanicolaou, priorizando o treinamento das equipes do Programa Saúde da Família (PSF), em municípios com cobertura inferior a 70\% de exames preventivos e com população superior a 10 mil habitantes 30 .

No entanto, a estimativa da razão entre exames citopatológicos cérvico-vaginais em mulheres de 25-59 anos e a população feminina nesta faixa etária para Minas Gerais no ano de 2007 foi de 0,2 (http://www.datasus.gov.br). Tal medida é utilizada para indicar a cobertura do exame de rastreamento na população-alvo, avaliando, de forma direta, a disponibilidade de ações básicas de prevenção e controle (educação para a saúde, captação e diagnóstico precoce) do câncer de colo de útero. As razões acima de 0,3 podem ser consideradas adequadas, na medida em que é preconizada a realização de um exame a cada três anos após dois resultados negativos de exames anuais 31 . Assim sendo, o valor estimado para o estado e acima referido se mostra ainda insatisfatório.

Neste contexto, cabe ressaltar que, em países desenvolvidos como Islândia e Finlândia, existem programas de rastreamento do câncer cervical organizados desde a década de 60, o que, em parte, explica a queda na mortalidade observada nestes países. Entretanto, estes países têm vivenciado um aumento na incidência de casos de câncer de colo de útero no início dos anos 90.
Mudanças no comportamento sexual são sugeridas como prováveis fatores responsáveis pelo mencionado aumento na incidência, que não é refletido na mortalidade devido, provavelmente, ao efetivo tratamento oferecido às pacientes acometidas pela doença 4,5. Além disso, deve-se considerar o fato de que, apesar do aumento da incidência observado para as faixas etárias mais jovens, os casos da doença têm sido diagnosticados em estádios mais iniciais na Islândia 5 .

Fatores sócio-econômicos têm sido apontados como importantes elementos associados à história natural desta doença. A melhora nos padrões de higiene foi considerada como fator relevante para a queda da mortalidade e da incidência do câncer de colo uterino na Escócia 8, e para a redução da incidência da doença na França ${ }^{32}$. Mudanças no comportamento sexual, principalmente relacionadas ao advento da AIDS, podem ter influenciado a queda na mortalidade, assim como contribuído para a queda na incidência da doença na França ${ }^{32}$. Para melhor entendimento dos achados verificados no presente estudo, deve-se levar em conta que o Estado de Minas Gerais exibiu considerável melhoria nos indicadores de saúde, de uma forma geral, no período considerado para este estudo, tais como: ampliação do número de domicílios com abastecimento de água e instalação sanitária; aumento do nível de escolaridade e de renda da população, entre outros.

Estudos têm demonstrado que avanços na abordagem terapêutica contribuem para a queda da mortalidade pela doença, tal qual a inclusão, a partir de 1999, da quimioterapia com cisplatina semanal ao tratamento tradicional radioterápico em tumores localmente avançados, que possibilitou a diminuição do risco de morte em, aproximadamente, $50 \%$ dos casos, devido à redução da recorrência local e à distância 33 . Neste sentido, cabe comentar, entretanto, que tal modalidade terapêutica ainda não está plenamente incorporada à assistência oncológica da rede pública do Brasil.

Deve-se ressaltar também a necessidade de levar em consideração, na interpretação dos achados anteriormente mencionados, as limitações inerentes a este estudo, a saber: a análise foi realizada para os dados de todo o Estado de Minas Gerais, sendo que a análise individualizada das diferentes regiões que compõem este estado poderia evidenciar diferenças marcantes; foram utilizados dados secundários, que podem exibir maior possibilidade de problemas na coleta e processamento, tais como erros de preenchimento do atestado de óbito e de codificação dos dados, bem como falhas na digitação, o que poderia comprometer os resultados; e, final- 
mente, o grande percentual de casos codificados como câncer de útero porção não especificada $(46,58 \%)$.

Finalmente, cabe destacar que a educação em saúde, o aprimoramento dos programas de rastreamento, a melhoria da qualidade da informação e, até mesmo, o aumento da abrangência dos RCBP devem ser considerados como importantes estratégias a serem adotadas para a redução da mortalidade e das repercussões físicas, psíquicas e sociais do câncer de colo uterino em nosso país. Apesar da tendência de redução da mortalidade por câncer cervical observada ao longo dos últimos 25 anos no Estado de Minas Gerais, ainda existe um importante caminho a ser percorrido para que se possa alcançar a cobertura preconizada e o acesso às medidas preventivas direcionadas ao controle da doença, especialmente no que se refere ao diagnóstico precoce.

\section{Resumo}

Foi avaliada a tendência da mortalidade por câncer cervical no Estado de Minas Gerais, Brasil, no período compreendido entre 1980-2005. Os dados demográficos e de mortalidade disponíveis na página da Internet do Departamento de Informática do SUS (DATASUS) foram utilizados para estimar a taxa de mortalidade por câncer de colo de útero e útero porção não especificada no local e período considerados. Foi realizada regressão linear, considerando-se a taxa de mortalidade como variável resposta e o ano do diagnóstico como variável explicativa. A mortalidade variou de 9,18/100 mil em 1980 para 5,70/100 mil em 2005. O modelo loglinear revelou redução da mortalidade por câncer cervical de cerca de 1,93\% ao ano na região e período avaliados ( $p=0,000$ ), sendo tal redução observada principalmente para os casos classificados como câncer de útero porção não especificada. A tendência observada de queda da mortalidade pela doença sugere melhoria no acesso a métodos de diagnóstico e tratamento oportunos e adequados em Minas Gerais nos últimos anos, embora a cobertura do rastreamento ainda seja insatisfatória.

Neoplasias do Colo do Útero; Saúde da Mulher; Mortalidade

\section{Colaboradores}

C. M. M. Alves participou da coleta de dados, análise e redação do artigo. M. R. Guerra e R. R. Bastos colaboraram na análise, redação e revisão crítica do artigo.

\section{Referências}

1. World Health Organization. Comprehensive cervical cancer control: a guide to essential practice. Geneva: World Health Organization; 2006.

2. Parkin DM, Bray F, Ferlay J, Pisani P. Global cancer statistics, 2002. CA Cancer J Clin 2005; 55:74-108.

3. Datta NR, Agrawal S. Does the evidence support the use of concurrent chemoradiotherapy as a standard in the management of locally advanced cancer of the cervix, especially in developing countries? Clin Oncol (R Coll Radiol) 2006; 18:306-12.

4. Anttila A, Pukkala E, Söderman B, Kallio M, Nieminen P, Hakama M. Effect of organised screening on cervical cancer incidence and mortality in Finland, 1963-1995: recent increased in cervical cancer incidence. Int J Cancer 1999; 83:59-65.

5. Sigurdsson K, Sigvaldason H. Effectiveness of cervical cancer screening in Iceland, 1964-2002: a study on trends in incidence and mortality and the effect of risk factors. Acta Obstet Gynecol Scand 2006; 85:343-9.

6. Vyslouzilova S, Arbyn M, van Oyen H, Drieskens S, Quataert P. Cervical cancer mortality in Belgium, 1955-1989: a descriptive study. Eur J Cancer 1997; 33:1841-5.

7. Arbyn M, Geys H. Trends of cervical cancer mortality in Belgium (1954-1994): tentative solution for the certification problem of unspecified uterine cancer. Int J Cancer 2002; 102:649-54.

8. Swerdlow AJ, Santos Silva I, Reid A, Qiao Z, Brewster DH, Arrundale J. Trends in cancer incidence and mortality in Scotland: description and possible explanations. Br J Cancer 1998; 77 Suppl 3:1-54. 
9. Comber H, Gavin A. Recent trends in cervical cancer mortality in Britain and Ireland: the case for population-based cervical screening. Br J Cancer 2004; 91:1902-4.

10. Tarone RE, Chu KC. Age-period-cohort analyses of breast-, ovarian-, endometrial- and cervicalcancer mortality rates for Caucasian women in the USA. J Epidemiol Biostat 2000; 5:221-31.

11. Liu S, Semenciw R, Probert A, Mao Y. Cervical cancer in Canada: changing patterns in incidence and mortality. Int J Gynecol Cancer 2001; 11:24-31.

12. Zheng TZ, Holford TR, Zheng MA, Chen Y, Liu W, Ward BA, et al. The continuing increase in adenocarcinoma of the uterine cervix: a birth cohort phenomenon. Int J Epidemiol 1996; 25:252-8.

13. Li H, Jin S, Xu H, Thomas DB. The decline in the mortality rates of cervical cancer and a plausible explanation in Shandong, China. Int J Epidemiol 2000; 29:398-404.

14. Wang PD, Lin RS. Age-period-cohort analysis of cervical cancer mortality in Taiwan, 1974-1992. Acta Obstet Gynecol Scand 1997; 76:697-702.

15. Ferlay J, Bray F, Pisani P, Parkin DM. GLOBOCAN 2000: cancer incidence, mortality and prevalence worldwide. Lyon: IARC Press; 2001. (IARC Cancer Base, 5).

16. Arrossi S, Sankaranarayanan R, Parkin DM. Incidence and mortality of cervical cancer in Latin America. Salud Pública Méx 2003; 45 Suppl 3: 306-14.

17. Kalakun L, Bozzetti MC. Evolution of uterine cervical cancer mortality from 1979 to 1998 in the State of Rio Grande do Sul, Brazil. Cad Saúde Pública 2005; 21:299-309.

18. Derossi AS, Paim JS, Aquino E, Silva LMV. Evolução da mortalidade e anos potenciais de vida perdidos por câncer cérvico-uterino em Salvador (BA), 1979-1997. Rev Bras Cancerol 2001; 47:163-70.

19. Fonseca LAM, Ramacciotti AS, Eluf Neto J. Tendência da mortalidade por câncer do útero no Município de São Paulo entre 1980 e 1999. Cad Saúde Pública 2004; 20:136-42.

20. Instituto Nacional de Câncer. Câncer no Brasil: dados dos registros de base populacional. v. 3. Rio de Janeiro: Instituto Nacional de Câncer; 2003.

21. Organização Mundial da Saúde. Manual da classificação estatística internacional de doenças, lesões e causas de óbitos - nona conferência de revisão. São Paulo: Centro Brasileiro de Classificação de Doenças em Português; 1985.
22. Organização Mundial da Saúde. Classificação estatística internacional de doenças e problemas relacionados à saúde, 10a revisão. São Paulo: Centro Colaborador da OMS para a Classificação de Doenças em Português; 1995.

23. Doll R, Cook P. Summarizing indices for comparison of cancer incidence data. Int J Cancer 1967; 2:269-79.

24. Holford TR, Zhang Z, McKay LA. Estimating age, period and cohort effects using the multistage model for cancer. Stat Med 1994; 13:23-41.

25. Curado MP, Edwards B, Shin HR, Storm H, Ferley J, Heanue $\mathrm{M}$, et al. Cancer incidence in five continents. v. IX. Lyon: IARC Press; 2007.

26. Levi F, Lucchini F, Negri E, Franceschi C, La Vecchia C. Cervical cancer mortality in young women in Europe: patterns and trends. Eur J Cancer 2000; 32:2266-71.

27. Wünsch Filho V, Moncau JE. Mortalidade por câncer no Brasil 1980-1995: padrões regionais e tendências temporais. Rev Assoc Méd Bras 2002; 48:250-7.

28. Silva IMR, Brenna SMF, Moriwaki OM, Mariani Neto C. Avaliação dos programas brasileiros para controle do câncer genital feminino. Rev Adm Saúde 2004; 6:97-102.

29. Bicalho SM, Aleixo JLM. O Programa "Viva $\mathrm{Mu}$ lher": programa nacional de controle do câncer de colo uterino e de mama. Revista Mineira de Saúde Pública 2002; (1). http://www.esp.mg.gov.br/ comunicacao/imagens_comunicacao/revista/ 1\%20-\%20Programa\%20Viva\%20Mulher.pdf.

30. Instituto Nacional do Câncer. Programa Nacional de Controle do Câncer de Colo de Útero e de Mama - Viva Mulher. http://www.inca.gov.br/conteu do_view.asp?id=140 (acessado em 22/Set/2008).

31. Ministério da Saúde. Portaria no ${ }^{\circ}$ 493, de 10 de março de 2006. Aprova a Relação de Indicadores da Atenção Básica - 2006, cujos indicadores deverão ser pactuados entre municípios, estados e o Ministério da Saúde. Diário Oficial da União 2006; 13 mar.

32. Remontet L, Estève J, Bouvier A-M, Grosclaude P, Launoy G, Menegoz F, et al. Cancer incidence and mortality in France over the period 1978-2000. Rev Epidemiol Sante Publique 2003; 51(1 Pt 1):3-30.

33. Monk BJ, Tewari KS, Koh W-J. Multimodality therapy for locally advanced cervical carcinoma: state of the art and future directions. J Clin Oncol 2007; 25:2952-65.

Recebido em 18/Dez/2008

Versão final reapresentada em 28/Abr/2009

Aprovado em 04/Mai/2009 